\title{
Can Statins Be Beneficial in COVID 19 Patients?
}

\author{
Mouin Jammal*, Moussa Riachy and Fady Haddad
}

Hotel Dieu de France, Saint Joseph University, Lebanon

*Corresponding author: Mouin Jammal, Hotel Dieu de France, Saint Joseph University, Lebanon, Tel: 9613812352

Covid-19 remains a novel pandemic with many controversies and variable clinical expression and severity. Diabetes and a pre-existing cardiovascular disease are the greatest risk factor for severe Covid-19. The overall case fatality rate of $2.3 \%$ in the general population increases to $7.3 \%$ in presence of diabetes and reaches $15 \%$ in presence of a pre-existing cardiovascular disease [1]. Age, smoking, arterial hypertension, hypercholesterolemia, diabetes and obesity predict a severe Covid-19.

MyD88 gene seems to be highly induced in SARSCoV infection. It's hallmark triad activates NFkB pathway, reduces type 1 interferon and increases inflammation [2,3]. Modified LDL cholesterol is absorbed via scatter receptors into cells. It forms crystals that activate NLRP3 inflammasome - caspase-1 activity increasing the release of IL-1b and IL-18 [4]. Oxidized LDL cholesterol binds to Toll-like family receptors which activates inflammation via MyD88 and NFkB pathway. This increases transcription of inflammatory cytokines such as IL6, IL-12, IL-27 \& TNF $\alpha$ [4].

Statins inhibit the MyD88 pathway maintaining normal levels during hypoxia or under stress [2]. The attenuation of NFkB increased the survival of SARS-infected transgenic mice [3]. The ability of statins to maintain MyD88 at normal levels may be protective for Covid-19 patients as the attenuation of NFkB inflammatory pathway reduces IL-6 and other inflammatory cytokines transcription. Furthermore, mortality was lower in COPD and influenza patients on moderate doses of statins compared to patients without statin therapy [5].

In our prospective cohort study, 65 patients hospitalized for severe Covid-19 received Pitavastatin known for few drug interaction, less muscular toxicity and bet- ter liver tolerability. Mean age was $53.1 \pm 18.3$ years and $60 \%$ were male. At database lock date, $80 \%$ of the patients were discharged with a median length of stay of 8 days, five remained in an isolation unit, one in ICU and only two patients were dead.

The ACE receptor seems to have also an important role in this disease. Thus, controlling cardiovascular risk factors tightly and exploring more the pathophysiology of Covid-19 will lead to a better comprehension of disease severity and variability and assure a better management.

\section{References}

1. Wu Z, McGoogan JM (2020) Characteristics of and important lessons from the Coronavirus Disease 2019 (COVID-19) outbreak in China: Summary of a report of 72314 cases from the Chinese Center for Disease Control and Prevention. JAMA 323: 1239-1242.

2. Yuan S (2015) Statins may decrease the fatality rate of middle east respiratory syndrome infection. mBio 6: e01120.

3. DeDiego ML, Nieto-Torres JL, Regla-Nava JA, Jimenez-Guardeño JM, Fernandez-Delgado R, et al. (2014) Inhibition of NF-kB-mediated inflammation in severe acute respiratory syndrome coronavirus-infected mice increases survival. J Virol 88: 913-924.

4. Cimato TR, Palka BA (2015) Effects of statins on TH1 modulating cytokines in human subjects. PeerJ 3: e764.

5. Frost FJ, Petersen H, Tollestrup K, Skipper B (2007) Influenza and COPD mortality protection as pleiotropic, dose-dependent effects of statins. Chest 131: 1006-1012. 